EPJ Web of Conferences 82, 01062 (2015)

DOI: $10.1051 /$ epjconf/20158201062

(C) Owned by the authors, published by EDP Sciences, 2015

\title{
Results of experimental studies operation chamber pump
}

\author{
Vyacheslav Bukhmirova and Maria Prorokova \\ Department of theory of heat engineering, Ivanovo State Power University, 153003 Ivanovo city, Russia
}

\begin{abstract}
At the moment there is a problem of high power consumption functioning pneumatic systems for bulk materials. A mathematical model of the motion of two-phase flow of fine materials with regard to the interaction of particles, uneven filling feeding pipeline and flow turbulence. The mathematical model can be used to calculate the motion of two-phase flow in pipelines, as well as in bulk pumps, subject to the incorporation of geometric design features and properties of the solid material in a stationary state. For adapting the mathematical model made industrial experiment and change the parameters obtained according to the time of discharge.
\end{abstract}

Pneumatic conveying system for bulk materials are widely used in building materials, metallurgy, as well as in a number of other industries. The most common are the pneumatic conveying system with chamber pumps. This paper presents the results of experimental studies of pneumatic systems intended to move the lime and cement (JSC "Bonola - Building Systems", Staraya Kupavna, Moscow reg.).

Experimental studies have been performed in order to obtain reliable data on the regime parameters of the chamber pumps and verify the adequacy of the mathematical model.

The goal of the experiment determination of the parameters of the pump pneumochamber:

- Air consumption, $\mathrm{V}, \mathrm{m} 3 / \mathrm{h}$;

- Air temperature, T, OC;

- Pressure at the inlet of the pump chamber, $\mathrm{P}_{\mathrm{H}}, \mathrm{kPa}$;

- Pressure in the chamber of the pneumatic pump, $\mathrm{P}_{\mathrm{K}}, \mathrm{kPa}$;

- Outlet pressure, $\mathrm{P}_{\mathrm{T}}, \mathrm{kPa}$.

Stage of preparation for the experiment involved the installation fittings in the pipeline for the installation pressure sensors. The scheme of the working section of the experiment is shown in Fig. 1. The experiment begins with the loading of the material in a pneumatic chamber $\mathbf{9}$ by a conduit $\mathbf{8}$. Compressed air is supplied to the air duct 7. The resulting two-phase flow in the chamber is removed on pipes 10. Measurement of flow and temperature of the compressed air is made using a submersible meter EE 7765 installed at a " $m$ " outlet pipe.

Pressure measurement is made by means of pressure sensors 6 type Oven ПД100-ДИ1,0-171-0,5 installed at points " $H$ ", " $k$ " and " $m$ " of the system (Fig. 1).

Collection of data was carried out by means of a discrete input-output Oven MК110-4ДН.4P 3 and automatic interface converter Oven AC4 2. Final signal processing performed by the program installed

\footnotetext{
${ }^{\text {a }}$ Corresponding author: buhmirov@tot.ispu.ru
}

This is an Open Access article distributed under the terms of the Creative Commons Attribution License 4.0, which permits unrestricted use, distribution, and reproduction in any medium, provided the original work is properly cited. 


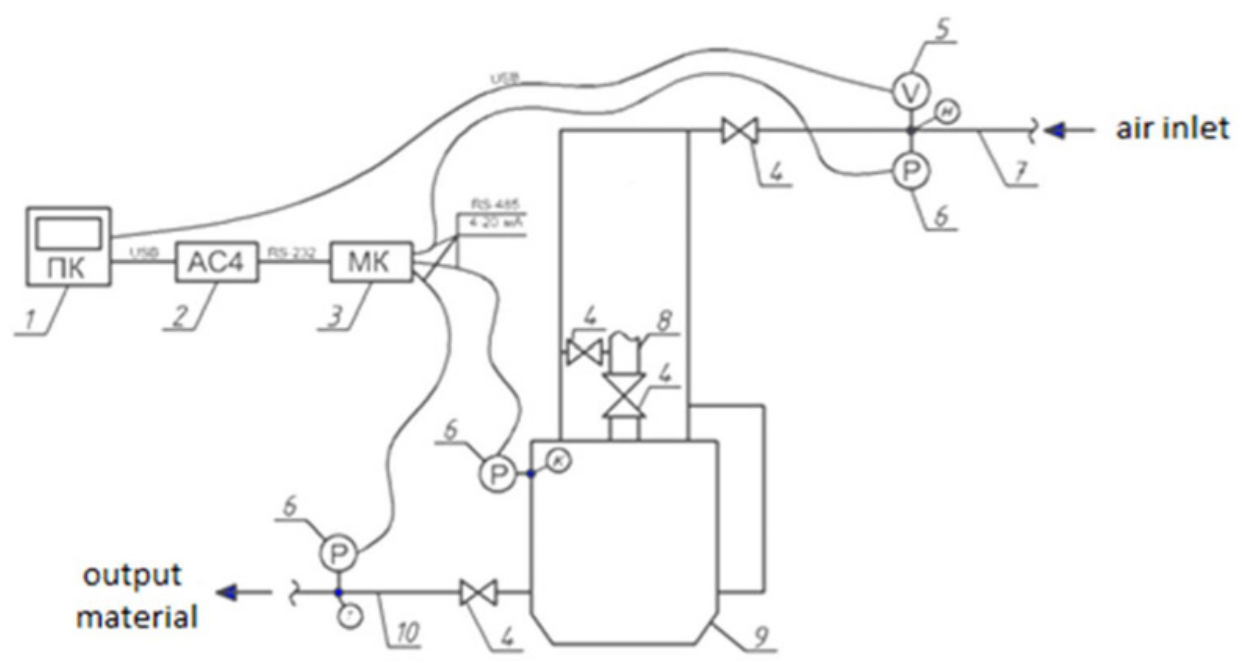

Figure 1. Diagram of the working area: 1 - personal computer; 2 - Automatic Interface Converter Oven AC4; 3 - module discrete input-output Oven MK110-4ДН.4P; 4 - Valves; 5 - meter submersible EE 776; 6 - Pressure sensor type Oven ПД100-ДИ1, 0-171-0,5; 7 - the air inlet; 8 - charging valve; 9 - a pneumatic chamber; 10 - the output material.

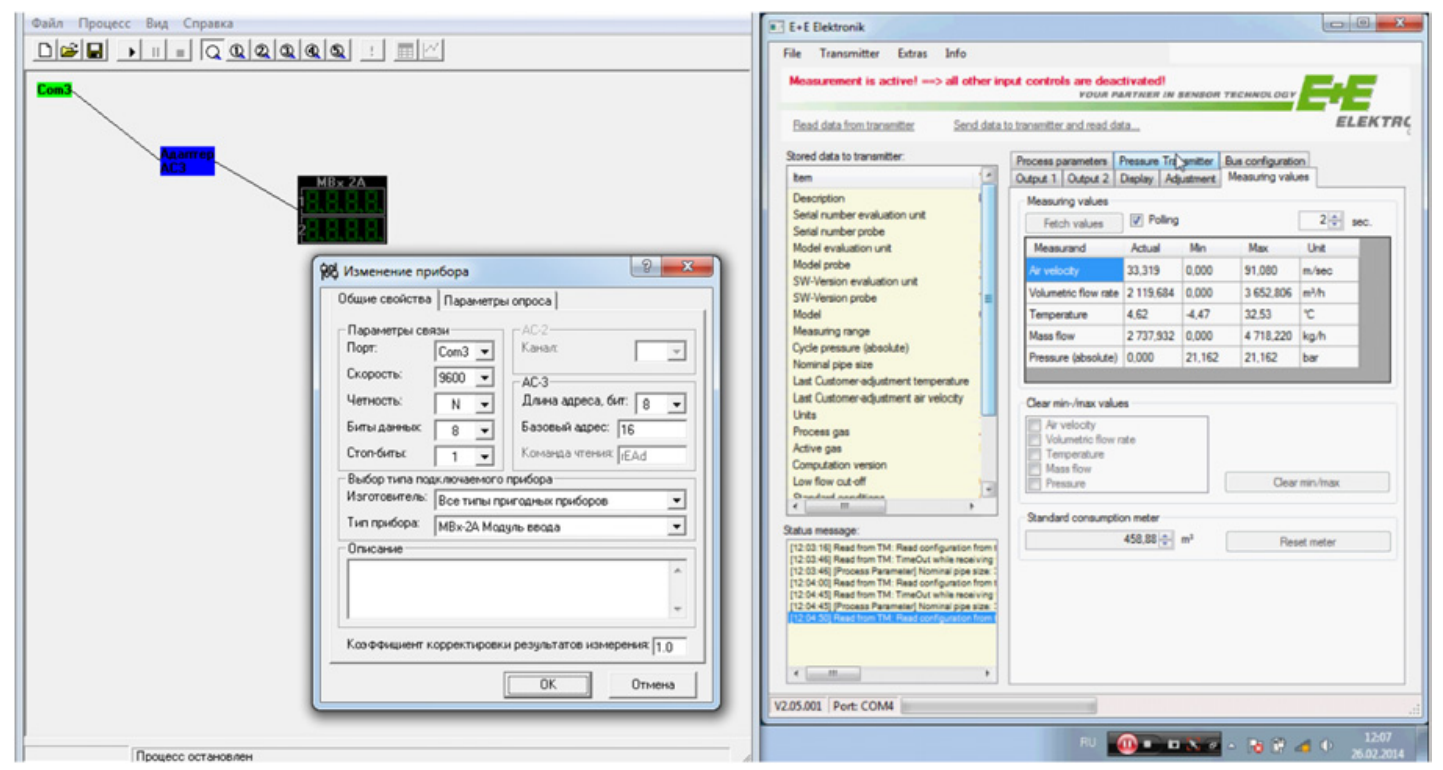

Figure 2. Data Collection System.

on the PC 1 to the data was used by SCADA - a software package designed for the development of or to provide real-time systems for the collection, processing, display and archiving information about the monitoring or management. As the SCADA system was selected OWEN PROCESS MANAGER (Figs. 2 and 3).

In the process of the experiment, data were obtained for six cycles pneumochamber pump. For each cycle are built unloading diagram which is a plot flow and temperature of the compressed air, and also to a pneumatic pressure in the pump chamber and the outlet. 


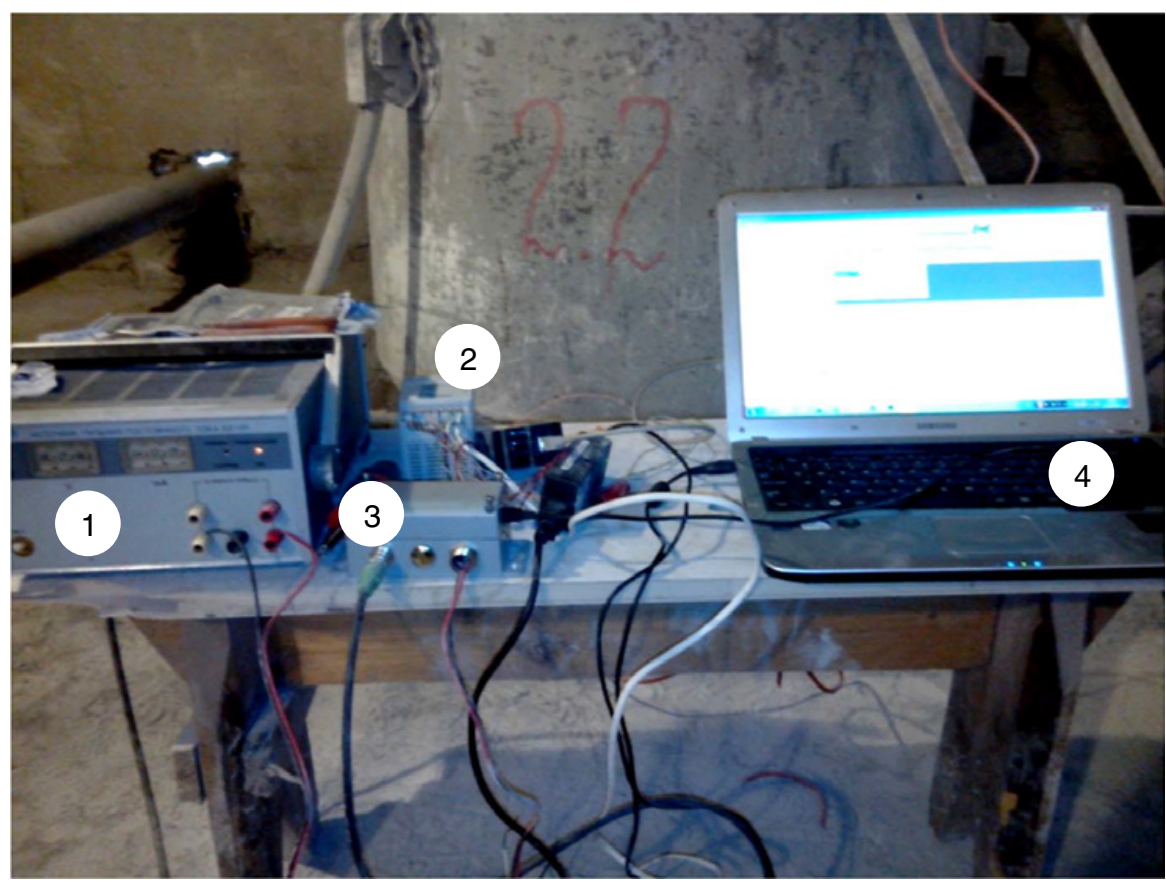

Figure 3. Device collect and process data: 1 - power supply unit; 2 - Digital Module input - output pressure sensors; 3 - module discrete input-output data flow meter; 4 - PC.

As a result of the experiment, the dependences of the pneumatic pressure in the chamber, flow and temperature during discharge. Averaged parameters discharge cycles of the pump chamber are shown in Fig. 4.

Analysis of Fig. 4 indicates that during the unloading process can be divided into three characteristic periods. In the first period dramatically increases air flow (its velocity) in the air line and set the pressure in the chamber pneumochamber pump type "Монжус." The duration of the first period of 2-6. Second period - mode stationary discharge. Pressure in the chamber of the pneumatic pump decreases monotonically; air flow rate varies slightly. In the second period is removed to $90 \%$ of the material. The duration of 43-52. The third period - the mode of residual discharge. Characterized by gradual reduction of air flow conveying bulk material. A pneumatic pressure chamber changes slightly.

The conclusions.

1. Industrial tests chamber pumps type "Монжус".

2. Experimental studies of the pneumatic installations performed by means of complex instrumentation based on microprocessor series sensors Oven ПД100-ДИ1,0-171-0,5 controllers Oven МК110-4ДН.4P and automatic interface converter Oven AC4, allowing real-time to record the parameters of the discharge cycle chamber pumps. Consumption of compressed air and its temperature measured submersible meter EE 776.

3. Get a unique experimental data on the change of regime parameters (flow rate, temperature and air pressure) pump discharge cycle pneumochamber brand "Монжус" НО-324MA.

4. The results of an experimental study of pneumopumps used for setting modes pneumatic systems and modernize the design pneumatic conveying systems. 


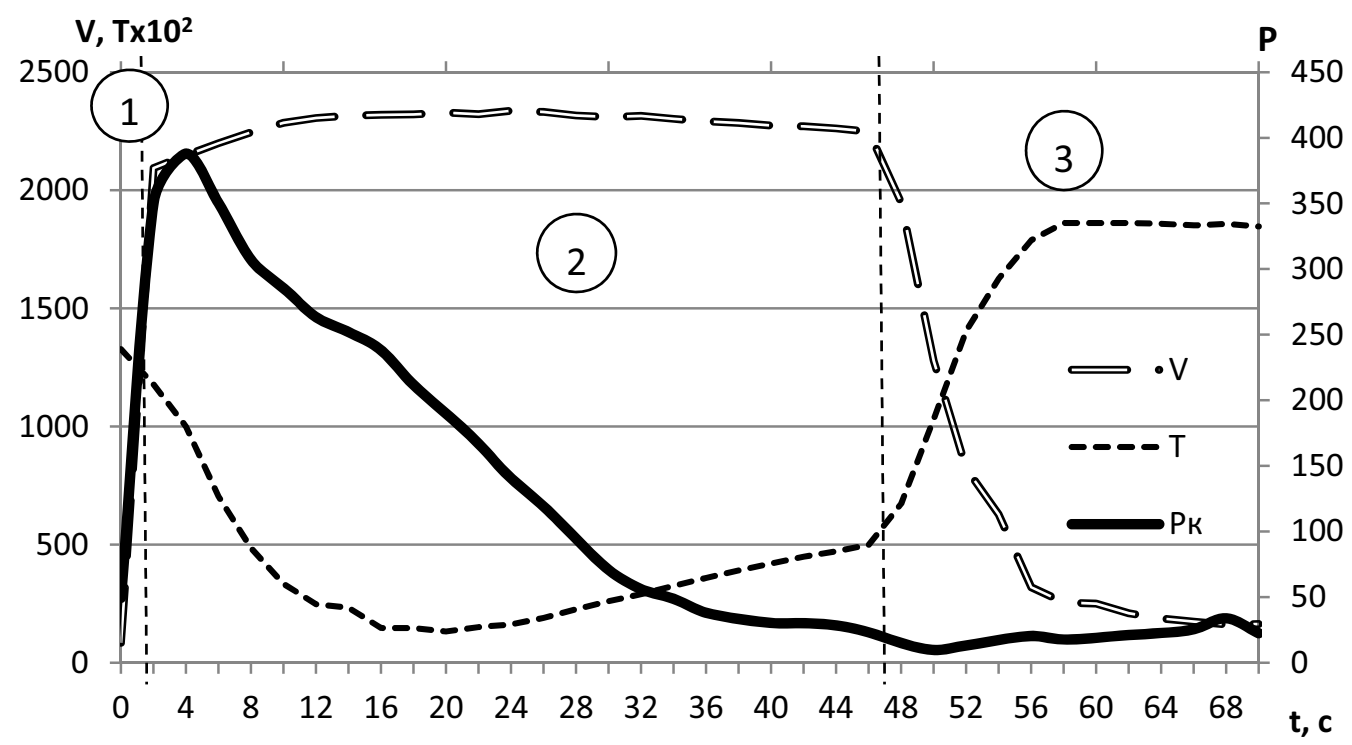

Figure 4. The average cycle parameters unloading chamber pump: $\mathrm{V}$ - air volume flow, $\mathrm{m}^{3} / \mathrm{h} ; \mathrm{T}-$ air temperature, ${ }^{0} \mathrm{C} ; \mathrm{P}_{\mathrm{K}}$ - the pressure in the chamber, $\mathrm{kPa}$.

5. Experimental data on changes in flow, pressure and temperature during discharge cycle pneumochamber pump used to verify the adequacy and improve the mathematical model of the pneumatic installations. 